\title{
Research on Influence of Sumsung Project on the International Logistics Development of Shaanxi Province
}

\author{
Dehong Xu \\ Xi'an International University, Xi'an Shaanxi, 710077, China
}

Keywords: Sumsung project, Reasons, Logistics of Shaanxi Province, Development influence

\begin{abstract}
As one of the world famous brands, Sumsung was founded in 1938, its Samsung Electronics includes heavy industry, securities, military industry and other industries, which drives the rapid development of economy. Sumsung project entered the high-tech zone of Xi'an city, Shaanxi Province in 2012, with first phase investment around 7 billion dollars, total estimated investment after the third phase is 30 billion dollars, Sumsung project drives the international logistics development of Shaanxi Province. This paper is to make a comparison of the international logistics scale of Shaanxi Province before and after of the entry of Sumsung project to drive the further development of its logistics and enlarge the scale.
\end{abstract}

\section{Overview and reasons of Sumsung project}

General situation of Sumsung project. When mentioned "Sumsung”, people firstly think of the Sumsung cellphone, as a Korean brand, Sumsung occupies half of Chinese cellphone market. Actually,Sumsung Group includes Samsung Electronics,Samsung Engineering,Samsung

SDS, Samsung Internet, Samsung Heavy Industries even Samsung Marine Insurance and other projects, it is the greatest business corporation, it is also a world famous brand. According to "The global top 2000 enterprises” of Forbes in2015, Sumsung Group is the second of the world, while according to the second quarter financial report that published by Sumsung, its profit of the second quarter is 5.75 trillion Korean Won. As one of the world corporate giants, the development trend of "Sumsung Group" influences the economic change of the whole world.

In 2012, Sumsung Company was founded in the high-tech zone of Xi'an in Shaanxi Province, April $10^{\text {th }}$ 2012, Samsung Electronics signed relevant agreement with Shaanxi Province, Xi'an Provincial Government and Xi'an High-tech Zone Administrative Committee, this strategic cooperation agreement includes important content of Samsung Electronics in China, mainly expressed as that the Korean Samsung Electronics settled its memory chips project in the High-tech Zone of Xi'an, and with the first phase investment of 7 billion dollars.September $12^{\text {th }}$, the 12 inches flash chips project started working, according to the report of Xi'an Evening News in May 2014, the Sumsung memory chips project officially put into production on May $9^{\text {th }}$ 2014, with over 1800 employees in the main production areas of Sumsung project, the factory construction has already finished, the Sumsung flash chips project was finished smoothly. The project construction of Sumsung Electronic in Shaanxi Province is the largest scale foreign investment project of electronic industry since open-door to the outside world, it is also the largest scale high-tech project of Shaanxi Province and Western China.With the continue development of this project construction, Xi'an High-tech Zone also attracted numerous domestic and foreign enterprises, including The United States Air Chemical, The East Beautiful Ken in South Korea and the domestic Huaxun Micro Electronics, which drives the systematization of Xi' an high-tech electronic industry.According to the statistical reports of Jingwei Network in 2015, until October 2015, Xi'an High-tech Zone attracted 88 supporting enterprises based on Sumsung ships project, with a total investment of 0.438 billion, at the same time, the number of attracted enterprises is continue growing, it was estimated to attract over 160 supporting enterprises to drive the rapid development of Xi'an.Meanwhile, according to the financial reports of Sumsung Group of August 2015, during this period, Sumsung Group has already realized over 10 billion accumulative value, this made China become the world industrial base for semiconductor side-by-side with the United States and South Korea, also drives the rapid economic 
development of Shaanxi Province, stimulates the internationalization and standardization of logistics industry.

Reason for the implementation of Sumsung project in Shaanxi Province. Production value of Sumsung Group in 2015 broke through 10 billion, at the same time, its encapsulation testing project also finished on April $14^{\text {th }}$ 2015, thus showed that Xi'an High-tech Zone has already formed a complete semiconductor production chain. The Sumsung semiconductor construction was started in 2012, at that time, cities like Beijing, Chongqing wanted to introduce in the Sumsung project, but at the last, Xi' an got it, the logistics environment of Shaanxi Province is an important reason. Specific reasons are as follows:

There are lots of Sumsung customers around Shaanxi Province, thus with concentrated logistics. The demand for Sumsung electronics around Shaanxi Province is very bug, there are lots of main customers of Sumsung electronics, the logistics here is integrated, radiate out from the important transportation junction, which can guarantee the rapid transportation and sales of Sumsung products.

Talent reserve in Shaanxi Province is very large and also with relevant talent preferential policies. High effective logistics system guarantees the introduction and development of talents of Shaanxi Province, large number of talents provide enough human resources for the construction of Sumsung project and help the stable development of enterprises.

As a logistics interaction, overall facilities of Shaanxi Province are good. The good-running electric power facilities and water supply plant guarantee the infrastructure the outside interaction of Shaanxi Province, which is good for the formation of semiconductor production line, it has ability to provide good transportation condition and communication equipment, thus it aroused the attention of Sumsung Group.

Relevant preferential policy provided by Shaanxi Provincial and Xi'an municipal government guaranteed the development of enterprises. The implementation of Developing Plan of Supporting Industries of Sumsung Project of Shaanxi Province in 2014, promoted the construction of Sumsung project, put forwarded the important requirements like "build one base, focus on two fields" and guided the communication of Sumsung company with the outer world, plan to construct a technical information industrial base that can radiate out to the whole world by making use of the convenient logistics condition of Shaanxi Province. Relevant preferential policy of Shaanxi Province is benefit to the development of Sumsung project and reduced the import and export tax, which further attracted Sumsung enterprises to build factory in Xi'an High-tech Zone.

\section{Logistics situation of Shaanxi Province before the entry of Sumsung project}

Logistics situation of Shaanxi Province. Modern logistics is a kind of service industry, not only including transportation fields, but also including industrial requirements like information transportation, warehouse management, as an important logistic interaction that connects Eastern and Western China, it occupies an important place in the logistic industry of China, in recent years, development of Shaanxi Logistics is more and more vigorous, but also has some problems. Firstly, from the development type of Shaanxi logistics, the logistics scale of Shaanxi Province is continuously increasing in 2011, total logistics value is 2.2615 trillion Yuan, accounts $7.3 \%$ of the GDP,among which national 2A logistics enterprises accounts $50 \%$ of the five provinces in North-west China, formed a logistics network of multiple ownership and service model, which drove the logistics development of Shaanxi Province. From our former research we know that one of the important reason of Sumsung's entry is the logistics, it is because that Shaanxi Province connects logistics of Central west, thus can promotes the rapid transportation of Sumsung products, thus the real reason for its entry.

Secondly, from the external supporting equipment of logistics industry in Shaanxi Province, in recent years, equipment for logistics in Shaanxi Province is increasingly perfecting, the development of transportation developed most rapidly, railway mileage in the Shaanxi Province was increased, highway mileage is also increased, it was increased by 22\% in 2010 than in 2009, density of highway is increased by $2.43 \%$, formed the one-day circle of surrounding cities, guarantee the day trips of 
major cities, this convenient transportation promoted the rapid development of logistics in Shaanxi Province, which will inevitably promotes the construction of Sumsung project, capital flow and product export enhances each other to promote the prosperous of Sumsung Group.

Thirdly, from the development of the third party logistics, the third party logistics means that part of enterprises use their own transportation to transport products to promote the development of their companies. For a simple example, as an excellent enterprise with third party logistics, 360 Buy promoted the development of the whole enterprise through self advertisement, self-sales and self-transportation, and further aggravate the competitive atmosphere of the logistics industry to realize the fairness, on the other hand, during the process of developing its own logistics, outsource $30 \%$ of its logistics business to relevant logistics enterprises can also promote the prosperous of logistics industry. Though as the logistics interaction of the Central East, logistics of Shaanxi Province also has lots of problems, the third party logistics is still at the introduction and promotion stage in the logistics development of Shaanxi Province, logistics outsourcing company only accounts less than $10 \%$ within the province, the whole development is slow.

At last, analyze the international logistics level pf Shaanxi Province, though it occupied an important position in China, but because lacking of relevant international brand and enterprises, the international logistics scale in Shaanxi Province was small before the entry of Sumsung Group, the logistics gross is small, cannot drive the products in Shaanxi Province to overseas, go to the world, the capital flow, product flow and information flow of the international logistics was less.

Problems exist in the logistics development of Shaanxi Province. It must overcome the following 5 problems to form a logistics internet that covers the whole Shaanxi Province to bring the logistics industry of Shaanxi Province to the world.First of all, the logistics scale of Shaanxi Province is small, its service level is low.Shaanxi logistics industry must improve the service level and mode that influenced by current system and concept,improve whole service level, stimulate the standardization and integration of logistics engineering to drive the development of the third party logistics,if Shaanxi Province wants to improve the development of international logistics industry.Secondly, reducing the logistics cost to drive the rapid development of international logistics development of Shaanxi Province must realize the problem of high logistics cost and improve logistics management method, use the advanced international cost calculation and control standard to reduce logistics cost and improve logistics efficiency.Thirdly, improve external condition of logistics in Shaanxi Province, though the transportation industry develops fast in recent years, its whole scale is still small and hard to meet the requirement of social and economic development, the utilization rate of highway in Shaanxi Province is low and distributes unevenly, the total highway in North Shaanxi and Shaanxi-Sichuan area is rare, transportation ability is low, which affects the further development and comprehensive coverage of logistics in Shaanxi Province.Fourthly, lack of professional talents to construct logistics enterprises, though with a large population, professional logistics talents in Shaanxi Province is less, which affects the professional development of logistics industry in Shaanxi Province. Fifthly, lack of funds to construct logistics enterprises, the whole fund amount is less, which affects the development of logistics in Shaanxi Province.

\section{Influence of developing Sumsung project on international logistics in Shaanxi Province}

Since the implementation of Sumsung project in Shaanxi Province, net profit of Sumsung project in 2015 has already broken through 10 billion, greatly drove the economic development of Shaanxi Province, brought in large number of professional talents, further speed up the development of logistics, mainly expressed in the following five aspects:

Sumsung project promotes investment input, promotes the construction of international logistics development in Shaanxi Province. The first phase investment of Sumsung project is 7 billion dollars, the estimated investment of the third phase is 30 billion dollar to promote the rapid development of Sumsung project, meanwhile, it will strengthen the whole economic level of Shaanxi Province,it also arouses Shaanxi Government's attention to highway and railway transportation, pay attention to the development of logistics enterprises, promotes the foreign trade of Sumsung Group by making use of the rapid logistics ability. The funds brought by Sumsung project solved the 
financial difficulty in logistics construction in Shaanxi Province and provided capital base for the logistics construction in Shaanxi Province.

Sumsung project attracted relevant enterprises into Shaanxi Province and formed an integrated logistics chain. According to report in October 2015, Sumsung project attracted 88 supporting enterprises to set in Shaanxi Province to construct technical park fir high-tech industry, this kind of supporting enterprises formed the integrated production mode, sell products all over the world through logistics network, which is good for the formation of integrated logistics chain. Promoting the better and rapid development of logistics enterprises based on guaranteeing its effectiveness and practicability and operation of the logistics.

Sumsung project attracted large number of technical talents into the High-tech Zone, promoted the construction of logistic industry. Logistics construction lacks of professional talents, the construction of Sumsung project attracted large number of highly-component talents into Shaanxi Province, large number of talents swarm into Shaanxi Province based on the good reputation and capital scale of Sumsung Group, and high welfare and high salary, which will bring Shaanxi Province a "talents spring" to promote the large-scale, integration and normalization development of logistics of Shaanxi Province.

The international brand of Sumsung ship promotes the logistics activities of Shaanxi Province. As a world brand, Sumsung Electronics has a good reputation, its entry in Shaanxi Province will promote the rapid development of economy of Shaanxi Province and promote the logistics in Shaanxi Province into the international trend, there is a large demand of Sumsung ships in the world, this will bring logistics of Shaanxi Province to be international and to make it more international and standardized.

Sumsung project promoted the international logistics of Shaanxi Province to make it play its pivotal role. As an important hub connects Central West of China, large number of western products are transported to the east and North China through Shaanxi Province, the western products can only be sold to the overseas through Shaanxi Province, through the implementation of Sumsung project, the hub function of Shaanxi Province can be realized and to promote our western products to go into the world.

\section{Conclusion}

In conclusion, as a world brand, Sumsung Group occupies an importance place in the world. Since Sumsung Electronics's entry of its memory ships into Xi'an High-tech Zone in 2012, this is the largest scale foreign investment project. Shaanxi Province is the important hub connecting Central and West, though the transportation scale is continuously extended in recent years, Shaanxi Province also has problems like small scale of logistics, lack of development funds and professional talents, while implementation of Sumsung project in Shaanxi Province is based on its important hub position and also promoted the large-scale, nationalization, standardization and integration development, promotes more talents resources, more funds input promotes the whole economy prosperous of Shaanxi Province. Therefore, the development of Sumsung project promoted the logistics development of Shaanxi Province, provided world Sumsung products to logistics of Shaanxi Province, promoted logistics of Shaanxi Province to use world standardized index, promoted the connection of domestic and overseas logistics and promoted the progress of international logistics of Shaanxi Province.

\section{Acknowledgments}

This paper is the social science fund project of Shaanxi Province, project name is Research on Influence of Sumsung Project on the International Logistics Development of Shaanxi Province. No.:13D246. 


\section{References}

[1] Shaanxi Logistics Guide. Shaanxi Science and Technology Press, 2010.

[2] Wang Rui. Research on the current Development Situation and Countermeasures of Shaanxi Logistics Industry.Xi'an University of Technology, 2011.

[3] Ding Wei; Zhang Liang; Li Jian; Analysis on the Construction of Axis - radial Modern Logistics Network.Soft Science in China, 2010(8):11-13

[4] Yang Guiling. Demand Analysis and Prediction of Logistics in Shaanxi Province. Chang'an University, 2011

[5] Xue Qing. Research on the Logistics Network Plan of Guanzhong Urban Agglomeration.Xi'an University of Technology, 2008.

[6] Zhou Tai. Wang Yaling. Evaluation on Regional Logistics Development Level based on Fuzzy Matter-element.Journal of Beijing Jiaotong University (JCR Social Science Edition), 2010(3). 